\title{
Magnetic signatures of plasma-depleted flux tubes in the Saturnian inner magnetosphere
}

\author{
N. André, ${ }^{1,2}$ A. M. Persoon, ${ }^{3}$ J. Goldstein,${ }^{4}$ J. L. Burch,${ }^{4}$ P. Louarn, ${ }^{5}$ G. R. Lewis, ${ }^{2}$ \\ A. M. Rymer, ${ }^{6}$ A. J. Coates, ${ }^{2}$ W. S. Kurth, ${ }^{3}$ E. C. Sittler Jr., ${ }^{7}$ M. F. Thomsen, ${ }^{8}$ F. J. Crary, ${ }^{4}$ \\ M. K. Dougherty, ${ }^{9}$ D. A. Gurnett, ${ }^{3}$ and D. T. Young ${ }^{4}$ \\ Received 13 April 2007; revised 11 June 2007; accepted 28 June 2007; published 21 July 2007.
}

[1] Initial Cassini observations have revealed evidence for interchanging magnetic flux tubes in the inner Saturnian magnetosphere. Some of the reported flux tubes differ remarkably by their magnetic signatures, having a depressed or enhanced magnetic pressure relative to their surroundings. The ones with stronger fields have been interpreted previously as either outward moving mass-loaded or inward moving plasma-depleted flux tubes based on magnetometer observations only. We use detailed multiinstrumental observations of small and large density depletions in the inner Saturnian magnetosphere from Cassini Rev. A orbit that enable us to discriminate amongst the two previous and opposite interpretations. Our analysis undoubtedly confirms the similar nature of both types of reported interchanging magnetic flux tubes, which are plasma-depleted, whatever their magnetic signatures are. Their different magnetic signature is clearly an effect associated with latitude. These Saturnian plasma-depleted flux tubes ultimately may play a similar role as the Jovian ones. Citation: André, N., et al. (2007), Magnetic signatures of plasma-depleted flux tubes in the Saturnian inner magnetosphere, Geophys. Res. Lett., 34, L14108, doi:10.1029/2007GL030374.

\section{Introduction}

[2] During the first orbits of the Cassini spacecraft around Saturn in 2004, anomalous plasma [Burch et al., 2005; Hill et al., 2005; Mauk et al., 2005] and magnetic field [André et al., 2005; Leisner et al., 2005] signatures have been identified and interpreted as evidence for centrifugally driven interchange motions, lending confidence to the hypothesis

\footnotetext{
${ }^{1}$ Research and Scientific Support Department, European Space Research and Technology Centre, European Space Agency, Noordwijk, Netherlands.

${ }^{2}$ Mullard Space Science Laboratory, University College London, Dorking, UK.

${ }^{3}$ Department of Physics and Astronomy, University of Iowa, Iowa City, Iowa, USA.

${ }^{4}$ Southwest Research Institute, San Antonio, Texas, USA.

${ }^{5}$ Observatoire Midi-Pyrénées, Centre d'Etude Spatiales des Rayonnements, Toulouse, France.

${ }^{6}$ Johns Hopkins University Applied Physics Laboratory, Laurel, Maryland, USA.

${ }^{7}$ NASA Goddard Space Flight Center, Greenbelt, Maryland, USA.

${ }^{8}$ Los Alamos National Laboratory, Los Alamos, New Mexico, USA.

${ }^{9}$ Blackett Laboratory, Imperial College, London, UK.
}

Copyright 2007 by the American Geophysical Union. 0094-8276/07/2007GL030374 that it is a common process in rapidly rotating magnetospheres like those of Saturn and Jupiter.

[3] The reported plasma signatures consisted of discrete hot plasma injection events accompanied by longitudinal magnetic gradient and curvature drift dispersion. They were observed both locally [Burch et al., 2005] and remotely [Hill et al., 2005; Mauk et al., 2005] by the Cassini Plasma Spectrometer (CAPS) and the Magnetospheric Imaging Instrument (MIMI). These signatures were consistent with expectations for the interchange of magnetic flux tubes containing dense and cold plasma with flux tubes containing tenuous and hot plasma that originated from farther out in the magnetosphere. However, the details of the resulting plasma circulation remain still elusive, particularly concerning the outward counterpart to those localized injections.

[4] The first magnetometer (MAG) data revealed that the Saturnian inner magnetosphere (inside $10 \mathrm{R}_{s}$ ) appears to be in an extremely dynamic state. André et al. [2005] presented a set of illustrations to support this picture, based on the identification of numerous short-duration sharp-bounded magnetic flux tubes whose content differ significantly from their surroundings. Particularly evident and abundant on the second orbit (Rev. A), some of these flux tubes were characterized by a depressed magnetic pressure, whereas a few others were characterized by an enhanced magnetic pressure. The former were observed between $6-8 \mathrm{R}_{s}$, within $1-2 \mathrm{R}_{s}$ of the equatorial plane, whereas the later were reported close to the time when Cassini crossed the Saturnian equatorial plane around $8 R_{s}$. Both types of magnetic signatures were interpreted as signatures of plasma-depleted flux tubes, returning planetward the magnetic flux that was carried outwards by mass-loaded flux tubes throughout the interchange process. On the other hand, Russell et al. [2006] interpreted interchanging flux tubes with enhanced magnetic pressure as agents for the outward transport of mass-loaded plasma to the tail of Saturn's magnetosphere. The studies of André et al. [2005] and Russell et al. [2006] were restricted to magnetometer data and pointed out the need for future multi-instrumental studies of these very interesting events.

[5] The present paper aims to detail further the magnetic signatures reported by André et al. [2005] in order to confirm their initial interpretation with the help of complementary measurements. We will show that plasmadepleted flux tubes can be characterized precisely by either depressed or enhanced magnetic field pressure signatures. We then discuss qualitatively some of the implications of 
these observations, in relation to the Jovian and terrestrial cases.

\section{Observations}

[6] Figure 1 represents simultaneous observations taken on October 28, 2004 by the MAG magnetometer, the CAPS ion (IMS) and electron (ELS) spectrometers, and the Radio and Plasma Wave Science (RPWS) instrument [Gurnett et al., 2004]. On this particular day, the Cassini spacecraft was embedded within the Saturnian inner magnetosphere, inside of 8.5 Saturn radii $\left(\mathrm{R}_{s}\right)$. The spacecraft was located above the Saturnian equatorial plane most of the time, except after 2000 UT when it crossed this plane, at $8.1 \mathrm{R}_{s}$. Closest approach occurred around $1000 \mathrm{UT}$ on this day, at $6.18 \mathrm{R}_{s}$.

[7] This multi-instrumental view complements the magnetometer observations given by of André et al. [2005, Figure 2 (right)]. The following list details the observations that are relevant to the present study:

[8] (1) Numerous small negative diamagnetic disturbances can be observed until 1600 UT (first panel of Figure 1). They represent average magnetic field changes of $1-2 \mathrm{nT}(1-2 \%$ of the background field) and last for several minutes. These cavities are observed at low magnetic latitudes, when the spacecraft is close to the outer latitudinal boundary of the plasmadisc (the plasma scale height is estimated to increase from $1 \mathrm{R}_{s}$ at $6 \mathrm{R}_{s}$ to nearly $2 \mathrm{R}_{s}$ at $8 \mathrm{R}_{s}$, cf. Persoon et al. [2006a]). After $1800 \mathrm{UT}$, large positive diamagnetic disturbances $(15 \%$ change of the background field) are observed when the spacecraft is very close to the equatorial plane, embedded in regions where the noise level of the magnetic field fluctuations is greatly enhanced.

[9] (2) A very clear upper hybrid resonance band can be seen above $10 \mathrm{kHz}$ until 1830 UT (second panel of Figure 1). This band can be used to deduce the total, local electron density. This technique was shown to be particularly well-adapted at Saturn inside of $8.4 \mathrm{R}_{s}$, with the upper hybrid emissions falling progressively below background noise levels beyond this distance [Persoon et al., 2005]. Electron cyclotron emissions can be observed above $1 \mathrm{kHz}$ from 1600 UT, when the spacecraft is very close to the equatorial plane. The emissions that drop below background noise levels after 1800 UT are the less intense, narrowband second order harmonic emission band. The intense primary emission band, which is spread over a broader frequency range, disappears or becomes patchy for only brief intervals.

[10] (3) The environment is fully pervaded by a twocomponent electron population (third panel of Figure 1). A cold population of tens of $\mathrm{eV}$ dominates the background plasma, whereas a hot population of hundreds to thousands of $\mathrm{eV}$ can be observed more episodically [Rymer et al., 2007]. Most of the time the hot electron population is associated with the discrete electron dispersion/injection events described by Hill et al. [2005] and Burch et al. [2005]. We note that the spacecraft potential is negative throughout this time interval.

[11] (4) Two distinct ion energy bands are noticeable on the IMS spectrogram (fourth panel of Figure 1). These approximately parallel bands correspond to the energy of water-group heavy ions and protons. They indicate, in particular, that these species have energies close to their corresponding corotational energy, proportional to their mass [Young et al., 2005]. Most of the electron dispersion/injection events have counterparts in ion observations.

[12] (5) The densities derived from CAPS ELS undergo smooth variations until 1800 UT (fifth panel of Figure 1). It appears difficult to distinguish radial variation from magnetic latitude variation during this particular orbit. A small but particularly well-marked density dropout is noticeable just before 0300 UT, whereas several strikingly large density dropouts can be observed after 1800 UT.

[13] On this large-scale view, it appears quite clearly that the most dramatic events are observed in all the data sets during the 1800-2100 time interval. Figure 2 details the multi-instrumental properties of some of these dramatic events (hereinafter referred to as events E) and Figure 3 details the properties of the well-marked event observed around $0300 \mathrm{UT}$ at $\mathrm{Z}=1.65 \mathrm{R}_{s}$ (hereinafter referred to as event D), previously reported by André et al. [2005, Figure 3] based on magnetometer data only.

[14] In both cases, events E and D correspond to magnetic flux tubes with properties that differ noticeably from those of their surroundings. The coordinated analysis of all observations provides us with deeper insights into the nature of the corresponding flux tubes:

[15] (1) Both events appear step-like, with very sharp boundaries. Their magnetic signatures (first panels of Figures 2 and 3) are mainly compressional. The change in the magnetic field direction (the magnetic components are not shown) in the case of events $\mathrm{E}$ is very small, whereas the magnetic signature of event $\mathrm{D}$ indicates that this particular flux tube is significantly more dipolar than its neighbors, similar to other events discussed by Leisner et al. [2005]. The magnetic pressure inside events E is significantly above ambient levels (magnetic field enhancement of $5 \mathrm{nT}$ in a background of $30 \mathrm{nT}$ ), whereas the magnetic pressure inside event $\mathrm{D}$ is below (magnetic field depression of $1 \mathrm{nT}$ in a background of $48 \mathrm{nT}$ ).

[16] (2) The upper hybrid frequency amplitude vanishes at the boundaries of events E, with a sudden and abrupt disappearance inside and re-appearance of the frequency band outside the injection events (second panel of Figure 2, e.g. 1905 UT). The weakness of the frequency line, close to the background noise level, makes it impossible to digitize densities from the upper hybrid resonance band in these events, but CAPS observations clearly reveal the presence of significant density dropouts inside these flux tubes. In the case of event $\mathrm{D}$, the upper hybrid frequency inside the flux tube is seen well below the upper hybrid frequency outside the flux tube (second panel of Figure 3, e.g. 0252 UT). This feature indicates that the corresponding flux tube is density-depleted.

[17] (3) The low-energy plasma population is severely depleted inside the corresponding flux tubes in both cases. It is replaced by a significantly hotter plasma population, compared to the ambient medium (third panels of Figures 2 and 3). The flux tubes are almost devoid of cold electrons $(\sim 10 \mathrm{eV})$, while the counts peak at significantly higher temperature, especially in the case of events E. A similar feature can be noticed in ion observations (fourth panel of Figures 2 and 3), albeit less clearly due to the patchy nature 

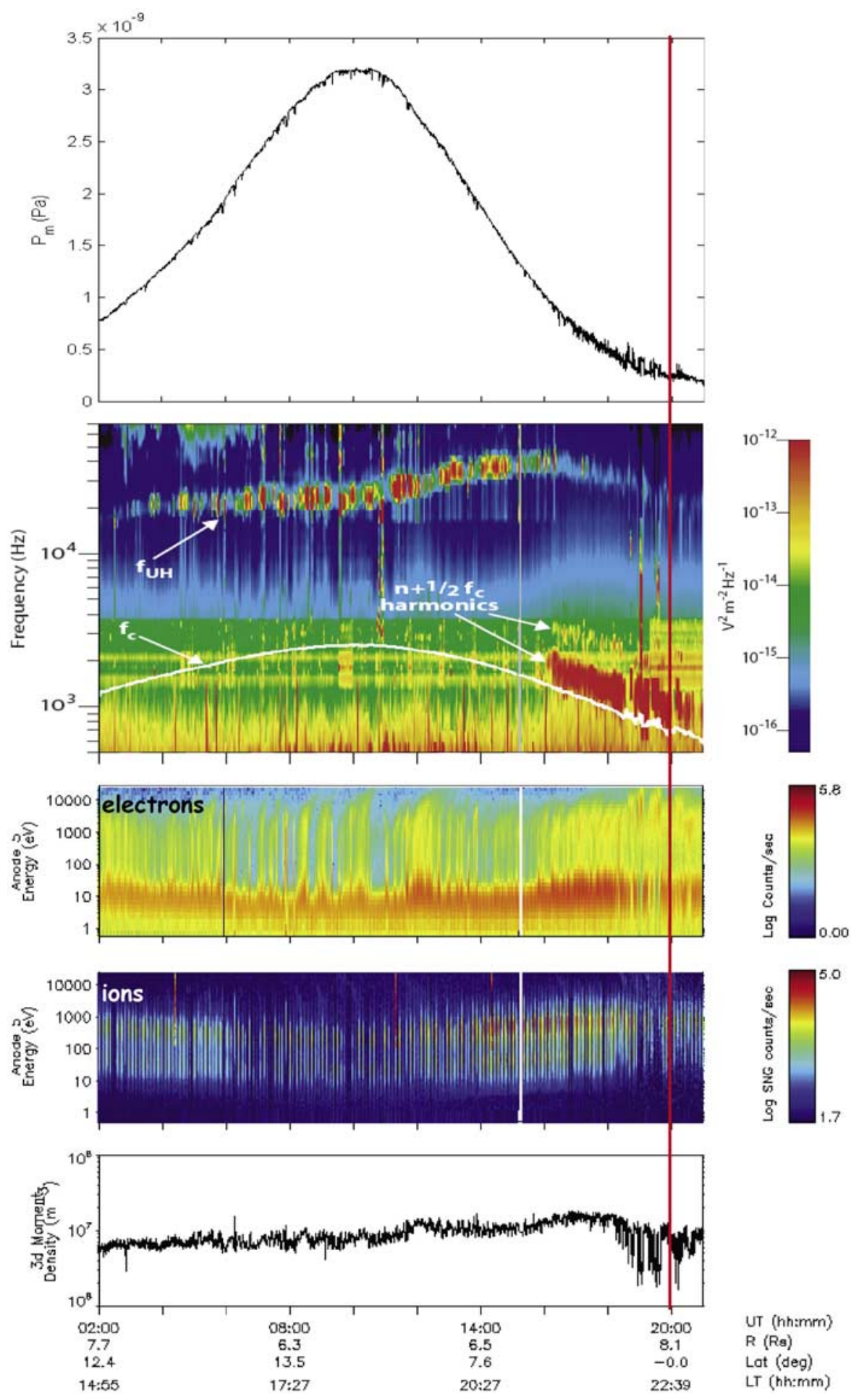

Figure 1. Overview of MAG, RPWS, and CAPS observations during the 0200-2100 time interval on October $28,2004$. From top to bottom: Magnetic pressure, color-coded frequency-time, electron and ion energy-time spectrograms, ELS electron density, versus time (in hours). Universal time (UT), radial distance (in $\mathrm{R}_{s}$ ), magnetic latitude (in degrees) and local time (LT) are given below. The red vertical line marks the crossing of the equatorial plane. The beaded appearance of the ion spectrogram is an artifact due to the rotation of the instrument.

of CAPS IMS observations associated with the rotation of the sensor.

[18] (4) The plasma densities derived from CAPS ELS confirm that the corresponding flux tubes contain clear and large density cavities in both cases (fifth panels of Figures 2 and 3 ), their densities being reduced by more than a factor 5 in the case of events E $\left(2 \cdot 10^{6} \mathrm{~m}^{-3}\right.$ compared to background densities of $10^{7} \mathrm{~m}^{-3}$ ) and by a factor 2 in the case of 

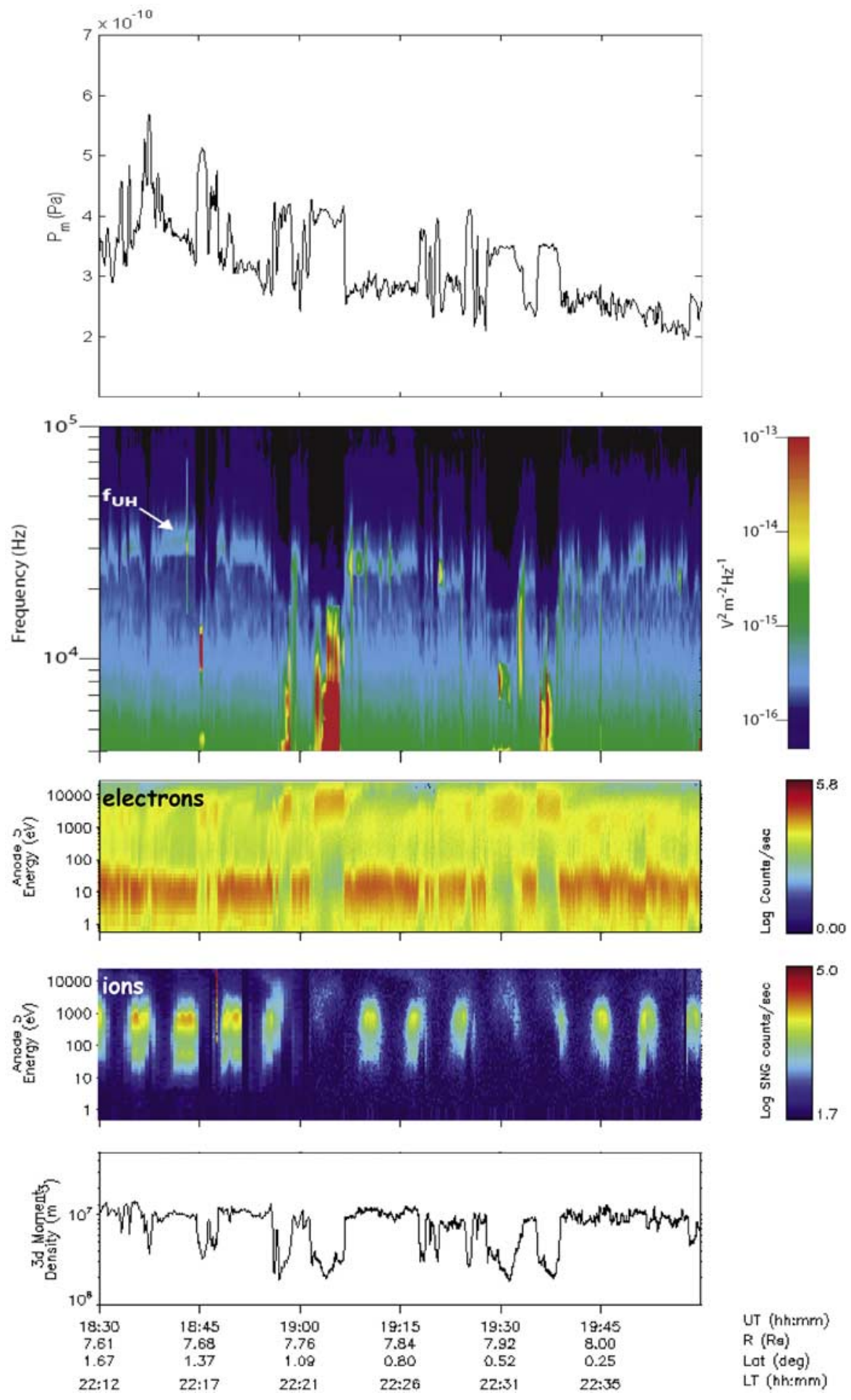

$$
\begin{aligned}
& \text { UT (hh:mm) } \\
& R \text { (Rs) } \\
& \text { Lat (deg) } \\
& \text { LT (hh:mm) }
\end{aligned}
$$

Figure 2. Zoom in on particular events observed during the 1830-2000 time interval on October 28, 2004.

event $\mathrm{D}$ (to $3.5 \cdot 10^{6} \mathrm{~m}^{-3}$ compared to background densities of $\left.7 \cdot 10^{6} \mathrm{~m}^{-3}\right)$.

[19] (5) An other flux tube with depressed magnetic pressure, similar to event $\mathrm{D}$, can be clearly identified around 0330 UT (first panel of Figure 3), where no evident depletion is observed in the derived plasma density (fifth panel of Figure 3). However, a clear drop in the upper hybrid frequency line (second panel of Figure 3) indicates that the corresponding flux tube is also density-depleted. Indeed, it can be sometimes difficult to identify density variations through the use of CAPS ELS data only, since the instrument does not measure the coldest part of the electron distribution when the spacecraft potential is negative. This 

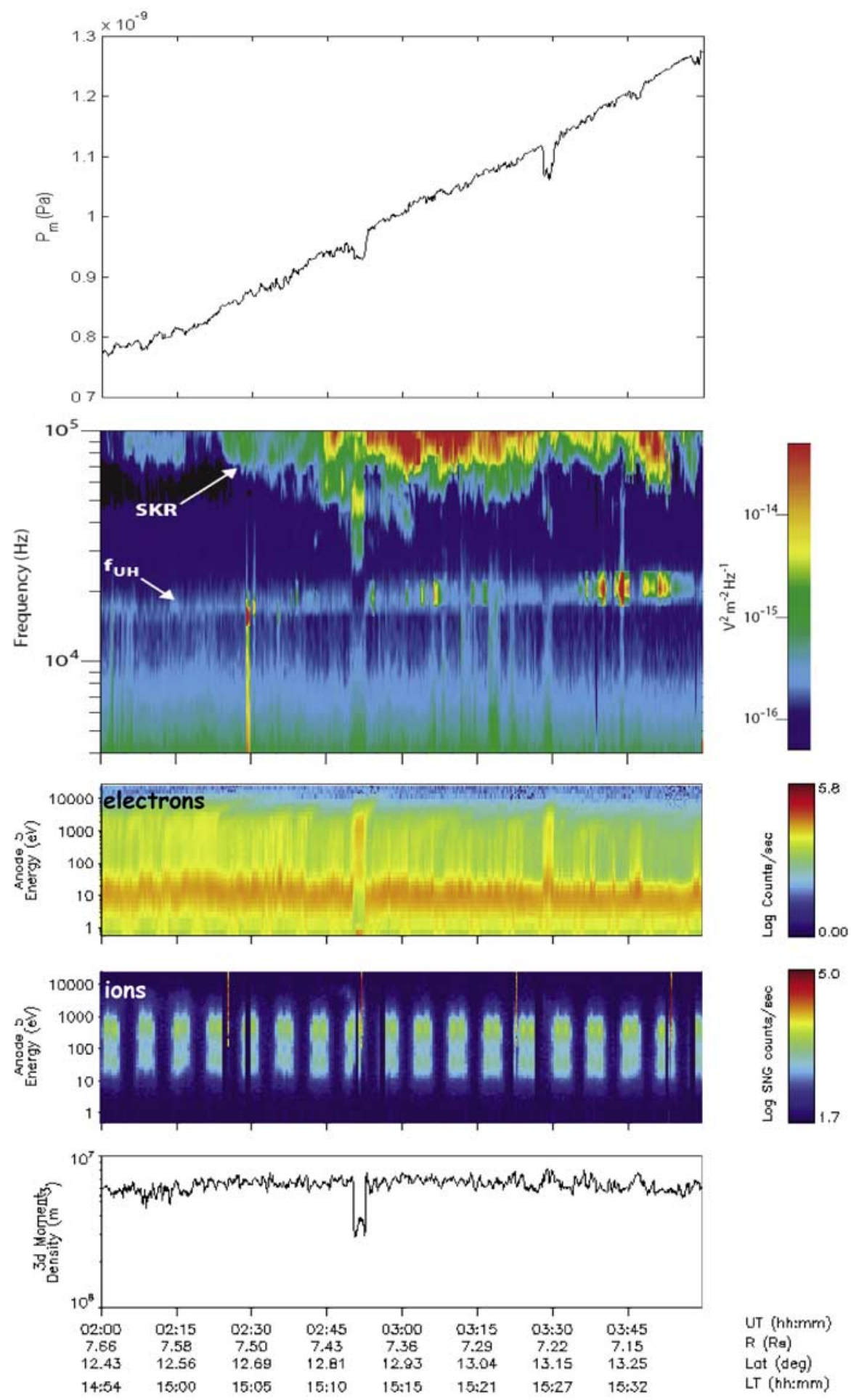

UT (hh:mm)
R (Rs)
Lot (deg)
LT (hh:mm)

Figure 3. Zoom in on particular events observed during the 0200-0400 time interval on October 28, 2004.

example illustrates the advantages of our multi-instrumental analysis.

[20] All the observational properties listed above undoubtedly confirm the initial interpretation of André et al. [2005] that both type of events correspond to plasmadepleted flux tubes. The parallel between both types of events is evident. They differ mainly by their magnetic signature (enhanced or depressed magnetic pressure) and also, in a quantitative way, by the magnitude of the associated density decrease and temperature increase.

\section{Discussion and Conclusions}

[21] In order to get significant statistical results, we surveyed all Cassini orbits in 2004-2005 and selected fifty clear events (inside $10 \mathrm{R}_{s}$ ) that corresponded to plasma- 


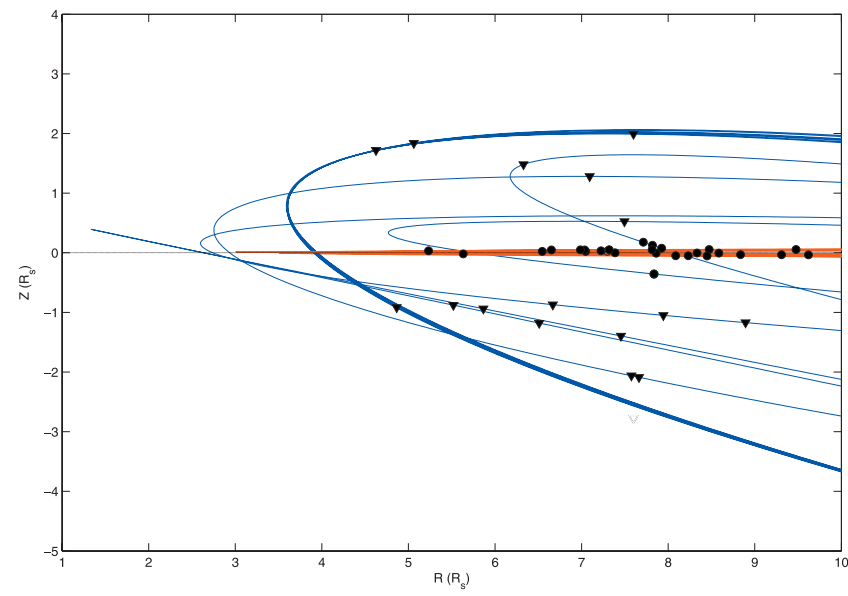

Figure 4. Cassini trajectories in the plane $(\mathrm{R}, \mathrm{Z})$ for years 2004-2005. R represents the radial distance to the planet and $\mathrm{Z}$ the distance to the magnetospheric equatorial plane, in $\mathrm{R}_{s}$. Events selected correspond to plasma-depleted flux tubes with depressed (triangles) and enhanced (circles) magnetic pressure, respectively.

depleted flux tubes. Our initial selection criterion was based firmly on the observation of visible density cavities (variations by at least a factor 2) associated with local injections of hot electron plasma (above $100 \mathrm{eV}$, CAPS ELS observations). We then carefully checked their corresponding ion signatures (CAPS IMS observations), the changes associated with the upper hybrid frequency emissions inside these events (abrupt disappearance lasting for several minutes, RPWS frequency-time spectrograms), and the behavior of their magnetic pressure (variations by at least $1 \mathrm{nT}$ of the magnetic field magnitude, MAG observations). Events reported by Russell et al. [2006] for Rev. 19 are included in our survey since they were found to satisfy our selection criterion. Local and therefore recent, hot electron plasma injections extend also in the tens of $\mathrm{keV}$ energy range, accessible to MIMI [Paranicas et al., 2007], as illustrated for Cassini Rev. 17 [cf. Paranicas et al., 2007, Figure 4]. These intense injections are also included in our survey and correlate with observations of plasma-depleted flux tubes with enhanced magnetic pressure (e.g., around 07:36 UT on October, 30 2005). Figure 4 displays the results of our survey in a meridian plane of the Saturnian magnetosphere. The most dramatic events, with enhanced magnetic pressure, are found to be concentrated very close to the equatorial plane, whereas events with depressed magnetic pressure are found off the equatorial plane at $\mathrm{Z}<2 \mathrm{R}_{s}$. Hence, their signature difference is clearly associated with latitude.

[22] In the absence of plasma bulk velocity measurements (currently under derivation, but not yet available), the following interpretation is based on the theoretical interpretation that plasma-depleted flux tubes are likely to return inwards in a rotation dominated magnetosphere. The main implication from the results displayed in Figure 4 is that inward plasma transport in the inner magnetosphere of Saturn occurs in localized events, characterized by plasmadepleted flux tubes associated with hot plasma injections, independently of their magnetic signature. As illustrated by our observations, outward plasma transport does not take the form of such discrete isolated events, and, on the contrary, evidence exists for this transport being consistent with a general plasma outflow [Burch et al., 2007] based on the observation of butterfly electron pitch-angle distributions in the background plasma. Therefore, the plasma circulation in the Saturnian magnetosphere appears to be very similar to the plasma circulation in the Jovian magnetosphere [Krupp et al., 2004; Russell et al., 2000; Russell, 2001]. In the terrestrial context, we note that Chen and Wolf [1993] carried out a model calculation of the shape of a pressure-balanced, underpopulated, inward moving flux tube (bubble) [cf. Pontius and Wolf, 1989], assuming its equatorial magnetic field exceeds that of its surroundings by a given factor. Owing to its more dipolar shape, they noted that the bubble's field line passes background field lines that cross the equatorial plane farther out. They pointed out that although the particle pressure is slightly less than that of its immediate neighbors in the equatorial plane, it exceeds that of its surroundings along nearly all of the length of the flux tube. Their model could be relevant to the Saturnian case, since a pronounced dipolarization in the equatorial plane (cf. events E) and a much rounder and dipolar shape off the equatorial plane (cf. event D or the events reported by Leisner et al. [2005]) are observed as plasma-depleted flux tubes convect towards Saturn.

[23] Our selection criterion is based initially on CAPS observations. In the future, a complete survey of smallerscale plasma-depleted flux tubes will be performed in correlation with RPWS measurements of the upper hybrid frequency emission band. Interestingly, these plasmadepleted flux tubes very often seem to be associated with a variety of electrostatic and electromagnetic plasma wave emissions, strongly confined within the boundaries of the flux tubes [Persoon et al., 2006b].

\section{References}

André, N., M. K. Dougherty, C. T. Russell, J. S. Leisner, and K. K. Khurana (2005), Dynamics of the Saturnian inner magnetosphere: First inferences from the Cassini magnetometers about small-scale plasma transport in the magnetosphere, Geophys. Res. Lett., 32, L14S06, doi:10.1029/ 2005GL022643.

Burch, J. L., J. Goldstein, T. W. Hill, D. T. Young, F. J. Crary, A. J. Coates, N. André, W. S. Kurth, and E. C. Sittler Jr. (2005), Properties of local plasma injections in Saturn's magnetosphere, Geophys. Res. Lett., 32, L14S02, doi:10.1029/2005GL022611.

Burch, J. L., J. Goldstein, W. S. Lewis, D. T. Young, A. J. Coates, M. K. Dougherty, and N. André (2007), Tethys and Dione: Sources of outward flowing plasma in Saturn's magnetosphere, Nature, 447, 833-835, doi:10.1038/nature05906.

Chen, C. X., and R. A. Wolf (1993), Interpretation of high-speed flows in the plasma sheet, J. Geophys, Res., 98, 21,409.

Gurnett, D. A., et al. (2004), The Cassini radio and plasma wave investigation, Space Sci. Rev., 114, 395.

Hill, T. W., A. M. Rymer, J. L. Burch, F. J. Crary, D. T. Young, M. F. Thomsen, D. Delapp, N. André, A. J. Coates, and G. R. Lewis (2005), Evidence for rotationally driven plasma transport in Saturn's magnetosphere, Geophys. Res. Lett., 32, L14S10, doi:10.1029/2005GL022620.

Krupp, N., et al. (2004), Dynamics of the Jovian magnetosphere, in Jupiter: The Planet, Satellites and Magnetosphere, Cambridge Univ. Press, New York.

Leisner, J. S., C. T. Russell, K. K. Khurana, M. K. Dougherty, and N. André (2005), Warm flux tubes in the E-ring plasma torus: Initial Cassini magnetometer observations, Geophys. Res. Lett., 32, L14S08, doi:10.1029/ 2005GL022652.

Mauk, B. H., et al. (2005), Energetic particle injections in Saturn's magnetosphere, Geophys. Res. Lett., 32, L14S05, doi:10.1029/2005GL022485.

Paranicas, C., D. G. Mitchell, E. C. Roelof, B. H. Mauk, S. M. Krimigis, P. C. Brandt, M. Kusterer, F. S. Turner, J. Vandegriff, and N. Krupp (2007), 
Energetic electrons injected into Saturn's neutral gas cloud, Geophys. Res. Lett., 34, L02109, doi:10.1029/2006GL028676.

Persoon, A. M., D. A. Gurnett, W. S. Kurth, G. B. Hospodarsky, J. B. Groene, P. Canu, and M. K. Dougherty (2005), Equatorial electron density measurements in Saturn's inner magnetosphere, Geophys. Res. Lett., 32, L23105, doi:10.1029/2005GL024294.

Persoon, A. M., D. A. Gurnett, W. S. Kurth, and J. B. Groene (2006a), A simple scale height model of the electron density in Saturn's plasma disk, Geophys. Res. Lett., 33, L18106, doi:10.1029/2006GL027090.

Persoon, A. M., N. André, D. A. Gurnett, W. S. Kurth, F. Akalin, A. J. Coates, and P. Canu (2006b), Plasma wave signatures of density-depleted flux tubes in Saturn's inner magnetosphere, Eos Trans. AGU, 87, Fall Meet. Suppl., Abstract SM23A-0288.

Pontius, D. H., Jr., and R. A. Wolf (1989), Transient flux tubes in the terrestrial magnetosphere, Geophys. Res. Lett., 17, 49.

Russell, C. T. (2001), The dynamics of planetary magnetospheres, Planet. Space Sci., 49, 1005.

Russell, C. T., M. G. Kivelson, W. S. Kurth, and D. A. Gurnett (2000), Implications of depleted flux tubes in the Jovian magnetosphere, Geophys. Res. Lett., 27, 3133.

Russell, C. T., J. S. Leisner, C. S. Arridge, M. K. Dougherty, and X. BlancoCano (2006), Nature of magnetic fluctuations in Saturn's middle magnetosphere, J. Geophys. Res., 111, A12205, doi:10.1029/2006JA011921.

Rymer, A. M., et al. (2007), Electron sources in Saturn's magnetosphere, J. Geophys. Res., 112, A02201, doi:10.1029/2006JA012017.
Young, D. T., et al. (2005), Composition and dynamics of plasma in Saturn's magnetosphere, Science, 307, 1262-1266, doi:10.1126/ science. 1106151 .

N. André, Research and Scientific Support Department, ESA/ESTEC, SCI-SM, Keplerlaan 1, NL-2200 AG Noordwijk, Netherlands. (nandre@ rssd.esa.int)

J. L. Burch, F. J. Crary, J. Goldstein, and D. T. Young, Southwest Research Institute, 6220 Culebra Road, P.O. Drawer 28510, San Antonio, TX 78238, USA.

A. J. Coates and G. R. Lewis, Mullard Space Science Laboratory, University College London, Holmbury St. Mary, Dorking, Surrey RH5 6NT, UK.

M. K. Dougherty, Blackett Laboratory, Imperial College, Exhibition Road, London SW7 2BZ, UK.

D. A. Gurnett, W. S. Kurth, and A. M. Persoon, Department of Physics and Astronomy, University of Iowa, 203 Van Allen Hall, Iowa City, IA 52242, USA.

P. Louarn, Observatoire Midi-Pyrénées, Centre d'Etudes Spatiales des Rayonnements, 9 avenue du Colonel Roche, F-31028 Toulouse, France.

A. M. Rymer, Johns Hopkins University Applied Physics Laboratory, 11100 Johns Hopkins Road, Laurel, MD 20723-6099, USA.

E. C. Sittler Jr., NASA Goddard Space Flight Center, 8800 Greenbelt Road, Code 612.2, Greenbelt, MD 20771, USA.

M. F. Thomsen, Los Alamos National Laboratory, P.O. Box 1663, Mail Stop D466, Los Alamos, NM 87545, USA. 